\title{
Análisis de una Plataforma Virtual 3-D Descentralizada para el Desarrollo de Simulaciones Educativas
}

\author{
Francisco A. Lizarralde y Constanza R. Huapaya \\ Universidad Nacional de Mar del Plata, Facultad de Ingeniería, Dpto. de Matemáticas, \\ Juan B. Justo 4302, Mar del Plata, Provincia de Buenos Aires-Argentina. \\ (e-mail: flizarra@fi.mdp.edu.ar, huapaya@fi.mdp.edu.ar )
}

Recibido Jun. 01, 2012; Aceptado Jul. 05, 2012; Versión final recibida Sep. 03, 2012

\begin{abstract}
Resumen
El objetivo del presente trabajo fue determinar si la plataforma Open Cobalt posee las características necesarias para implementar simulaciones interactivas en un ambiente colaborativo. La utilización y desarrollo de recursos como Open Cobalt, una herramienta para crear mundos virtuales, presentan un interesante campo de investigación, ya sea desde la perspectiva tecnológica como desde la educativa. Se eligió analizar esta plataforma principalmente porque posee un esquema de comunicaciones descentralizado y por estar implementada en un lenguaje de programación dinámico. Se estableció un conjunto de características a analizar de la plataforma y en base a los resultados obtenidos de las pruebas realizadas se concluye que la misma posee los recursos necesarios para generar simulaciones interactivas en un ambiente colaborativo.
\end{abstract}

Palabras clave: ambientes virtuales de aprendizaje, Open Cobalt, simulación, mundos virtuales

\section{Analysis of a Decentralized 3-D Virtual Platform for Educational Simulations Development}

\begin{abstract}
The aim of this work was to determine if the Open Cobalt platform has the necessary features to implement interactive simulations in a collaborative environment. The use and development of resources such as Open Cobalt, a tool for creating virtual worlds, present an interesting research field, from the technological perspective as well as from the educational point of view. This platform was selected for analysis mainly because it has a decentralized communications scheme and it is developed in a dynamic programming language. A set of features of the platform was chosen to analyze and based on the tests results it is concluded that Open Cobalt has the necessary resources to generate interactive simulations in a collaborative environment.
\end{abstract}

Keywords: virtual learning environments, Open Cobalt, simulation, virtual worlds 


\section{INTRODUCCIÓN}

Los Ambientes Virtuales de Aprendizaje o VLES (Virtual Learning Environments) representan actualmente una excelente herramienta para la administración de contenidos y recursos educativos. Desde cursos específicos en la modalidad de educación a distancia, hasta sistemas completos de blended-learning (Modalidad de aprendizaje en la que se conjuga la enseñanza mediada o a distancia y la presencial) y de e-learning que incluyen mecanismos de evaluación y asistencia personalizada a los alumnos.

Sin embargo, a pesar de su diversidad, la mayoría de estos ambientes comparte una característica en común, una interfaz de usuario bidimensional, basada principalmente en la metáfora de escritorio. Esta interfaz, aunque muy exitosa por cierto, presenta ciertas limitaciones en lo que respecta a la representación de ciertos contenidos y a la posible interacción con los mismos. En un espacio bidimensional, como un pizarrón o una página web se suele apelar a la utilización de símbolos, gráficos o diagramas, para expresar conceptos e ideas que exceden las posibilidades de representación de este medio. En cambio en un ambiente virtual tridimensional e interactivo, las alternativas de representación de contenidos aumentan considerablemente.

Frecuentemente se observa que los estudiantes de Ingeniería experimentan cierta dificultad para modelar los sistemas dinámicos y de control. Les resulta particularmente difícil visualizar el movimiento y la respuesta dinámica de los sistemas físicos. Los estudiantes suelen percibir a los conceptos, en particular a los relacionados con los sistemas dinámicos, como un conjunto de fórmulas matemáticas abstractas. Esto se debe a que las representaciones estáticas provistas por medios tradicionales como los libros de texto o el pizarrón resultan insuficientes para mostrar la respuesta dinámica de un sistema (Kypuros y Connolly, 2005).

Los entornos virtuales 3-D permiten construir y manipular tanto objetos, como modelos y representaciones metafóricas, lo cual incentiva a los estudiantes a involucrarse de una manera diferente con los temas y de esta forma mejorar su aprendizaje. El soporte pedagógico en el que se sustentan está basado principalmente en el paradigma constructivista, en el cual el estudiante posee un rol activo en su aprendizaje al relacionar el conocimiento asimilado con el nuevo que va descubriendo (Huang et al., 2010).

Es decir, la sensación de presencia en primera persona en los ambientes virtuales genera nuevos aprendizajes, pues ampliaría las formas de adquirir conocimiento en la interacción y transformación de ideas abstractas en representaciones perceptibles a través de objetos virtuales (Fredes et al. , 2012). Es así que ambientes virtuales como Second Life, creados inicialmente con una finalidad recreativa y de entretenimiento para el público en general, se han transformado actualmente en objeto de estudio para investigadores en educación y en una atractiva plataforma de enseñanza para muchas instituciones educativas (Warburton, 2009).

Bryn Holmes introduce un nuevo enfoque del aprendizaje (Holmes et al., 2001), al que denomina Constructivismo Comunal en el que los estudiantes no solo construyen su propio conocimiento (Constructivismo), como resultado de la interacción con su entorno (Constructivismo Social), sino que participan activamente en el proceso de construcción de conocimiento para su comunidad de aprendizaje. Es decir, los principios del Constructivismo Comunal sostienen que los estudiantes investigan y trabajan para crear artefactos de aprendizaje que serán usados y mejorados por futuros estudiantes (Girvan y Savage, 2010) y para lograr tal cometido necesitan contar con medios de comunicación e interacción adecuados.

La infraestructura de comunicaciones de la mayoría de los entornos virtuales 3-D actuales utiliza un esquema centralizado, en el que los usuarios se conectan a un servidor central por medio de un programa cliente, denominado visualizador (viewer). De este modo los contenidos se encuentran almacenados principalmente en dicho servidor, el cual se encarga además, de sincronizar la comunicación y el intercambio de contenidos entre los estudiantes. 
Un esquema diferente, muy utilizado en los videojuegos en red y en las redes de intercambio de archivos, es el conocido como Peer to Peer (P2P o redes de pares), el cual no posee un servidor central, sino que cada integrante de la red actúa a la vez como cliente y servidor de contenidos.

Sobre este modelo de comunicaciones está basado un proyecto de código abierto desarrollado en la Universidad de Duke, denominado Open Cobalt, el cual promueve la creación de mundos virtuales sobre arquitecturas distribuidas. Las conexiones simultáneas entre pares a través de Internet van creando verdaderas "comunidades" totalmente dinámicas en cuanto a su topología y cantidad de integrantes (Chun-Hong y Ming, 2008). De esta forma, los usuarios no se encuentran todos conectados directamente entre sí, sino que se van creando y modificando en forma dinámica, "grupos" de usuarios, en función de los contenidos que deseen compartir. Esto permite además, un mejor aprovechamiento del ancho de banda existente y brindar una velocidad de respuesta razonable a un número creciente de usuarios (McGeer y et al., 2006). Por otra parte, desde un punto de vista económico, este esquema sería bastante prometedor ya que permitiría desarrollar mundos virtuales interconectados con un costo relativamente bajo, pues no sería necesario mantener grandes servidores para proveer la interconexión de los espacios de los usuarios y el almacenamiento de contenidos (Lombardi y Lombardi, 2010).

\section{ANTECEDENTES DE OPEN COBALT}

Open Cobalt es un entorno tridimensional colaborativo desarrollado íntegramente en Squeak (Ingalls et al. 1997), (Guzdial, M. 2001) una versión moderna de Smalltalk. Smalltalk (Goldberg y Robson, 1989) es un lenguaje de programación orientada a objetos puro, creado en los laboratorios de investigación de Xerox, por el equipo de investigación a cargo del Dr. Alan Kay (Sproull, 2010), quien fuera galardonado con el Turing Awards en 2003 por su gran aporte a la informática. El objetivo principal de Smalltalk era lograr un entorno capaz de expandir la creatividad a través de un lenguaje orientado a la experimentación, creación e investigación.

En el año 2001, el Dr. Alan Kay junto con el Dr. David A. Smith iniciaron un proyecto denominado Croquet (Smith, 2003), cuyo objetivo era crear una plataforma de experimentación y generación de espacios virtuales basada en un nuevo paradigma de interfaces de usuario. Según Alan Kay, "Croquet fue construido para responder a una sencilla pregunta. Si tuviéramos que crear un nuevo sistema operativo y su interfaz de usuario, conociendo lo que hoy conocemos, ¿Hasta dónde podríamos llegar? ¿Qué tipo de decisiones podríamos tomar hoy, que hubiéramos sido incapaces incluso de considerar hace 20 o 30 años, cuando se crearon inicialmente los sistemas operativos actuales?"

A partir de esta premisa, varias universidades, institutos de investigación y empresas privadas se reunieron para aportar los recursos humanos y financieros necesarios para este proyecto. Este esfuerzo conjunto permitió en el año 2006 desarrollar una versión beta del Croquet SDK (Software Development Kit), cuya infraestructura tecnológica comenzó a utilizarse exitosamente en empresas comerciales como QWAQ para construir aplicaciones colaborativas privadas. Lamentablemente gran parte de este código desarrollado bajo licencias más restrictivas no pudo distribuirse como código abierto. Sin embargo, en el año 2008, un equipo de la Universidad de Duke, liderado por el Dr. Julián Lombardi, uno de los principales arquitectos de software de Croquet, retomó su desarrollo, ahora bajo el nombre de Open Cobalt, transformándolo en una plataforma que actualmente se distribuye bajo una licencia de código abierto (Open Cobalt License, 2008).

Esta breve reseña histórica acerca de la evolución de Open Cobalt resulta imprescindible para comprender algunas de las razones que nos Ilevaron a elegir a Open Cobalt como objeto de análisis en el presente trabajo. Si bien esta elección se debe en gran medida a sus características técnicas particulares, como por ejemplo, que se trata de una aplicación de código abierto, multiplataforma y descentralizada, existe un aspecto que nos parece de particular importancia. El objetivo primordial tanto de Open Cobalt como de los proyectos que le precedieron y le dieron origen, ha sido facilitar la expresión de ideas, promover la experimentación y proveer de los medios necesarios para un mejoramiento del aprendizaje. 
En el presente trabajo se analizará a la plataforma Open Cobalt para comprobar si es factible su utilización para implementar simulaciones interactivas en un ambiente distribuido. Para este cometido se estableció un conjunto de requerimientos mínimos a cumplir y se analizaron los resultados obtenidos.

El presente artículo está organizado de la siguiente manera: en la sección se presenta la metodología utilizada, la cual consistió en establecer un conjunto de características técnicas necesarias para lograr la implementación de simulaciones interactivas. Posteriormente se muestran los resultados obtenidos al evaluar cada una de ellas. Y finalmente se presentan las conclusiones.

\section{METODOLOGÍA}

La implementación de simulaciones en un ambiente de aprendizaje colaborativo requiere que la plataforma virtual 3-D sobre la que se desarrolle cumpla ciertos requisitos técnicos mínimos. Por esta razón establecimos un conjunto de características a evaluar y creamos pruebas y prototipos en Open Cobalt con los que buscamos valorar su desempeño. Las características consideradas fueron: Fidelidad de la representación, Navegabilidad e interacción con el entorno, Comunicación entre usuarios, Modelos 3-D y contenidos multimedia, Integración de aplicaciones y Entorno de programación.

La fidelidad de la representación es indispensable para lograr una experiencia "espacial" creíble, es decir, para que el usuario tenga la sensación de estar dentro de ese espacio interactuando con todos los elementos que allí se encuentran. Las tarjetas de video actuales poseen una gran potencia de procesamiento, lo que les permite manejar representaciones gráficas de gran calidad, texturas y efectos de iluminación con gran velocidad y en forma prácticamente autónoma, lo cual resulta imprescindible para lograr una representación dinámica convincente. A su vez, los objetos del entorno, así como las representaciones de los usuarios (avatars) necesitan comportarse consistentemente. Es decir, el usuario espera que dentro del entorno se cumplan ciertas "leyes físicas", por ejemplo, que la intensidad de las fuentes sonoras presentes, varíe de acuerdo a la posición y distancia que lo separa de las mismas (sonido espacial).

Por navegabilidad e interacción con el entorno, nos referimos a los mecanismos utilizados para recorrer los espacios y para modificar ciertos atributos, que afectan tanto al aspecto visual como a su comportamiento, así como también, la creación y manipulación de los objetos contenidos en el mismo. Esta interacción, generalmente se realiza por medio de dispositivos de señalamiento estándar (mouse, trackballs, etc.) y combinaciones de teclas, aunque en la medida que su costo disminuya probablemente se irá generalizando la utilización de dispositivos de navegación con acelerómetros incorporados (Wii), interpretación de movimientos (Kinect), o la interacción con los objetos del entorno por medio de interfaces táctiles (Dataglove).

En lo que respecta a la comunicación entre usuarios, hay dos aspectos a considerar, la comunicación oral-escrita y la gestual. La comunicación oral puede lograrse por medio de sistemas de voz a través de Internet, o VolP (Voice over Internet Protocol) y la comunicación escrita por medio de herramientas de mensajería instantánea, o IRC (Internet Relay Chat) integradas en la misma plataforma. Por otra parte, una característica propia de los mundos virtuales es la incorporación de la comunicación gestual, la cual se logra dotando a las representaciones visuales de los usuarios (avatars) de un cierto conjunto básico de movimientos o gestos.

Los modelos 3-D y los contenidos multimedia son recursos que producen una singular sinergia cuando se incorporan a los mundos virtuales. En el primer caso nos referimos a figuras, vehículos, edificios, etc. y en el segundo caso, se trata de fotografías, archivos de audio y video. En todos los casos para poder integrarlos como recursos pedagógicos, se requiere que el entorno tenga la capacidad de cargarlos y reproducirlos adecuadamente. 
Por otra parte, para que un entorno virtual no sea una aplicación aislada es conveniente que sea posible integrar aplicaciones externas dentro del entorno o al menos contar con la posibilidad de ejecutarlas aunque sea de forma remota. Esto permitiría a los alumnos la utilización de navegadores de Internet, planillas de cálculo, editores de texto, etc. sin necesidad de salir del mundo virtual.

Por último, los procesos de aprendizaje y experimentación, como las simulaciones, son más efectivos y motivadores si los mismos pueden ser modificados y compartidos con otros estudiantes (Leask y Younie, 2001). Para lograr este cometido, es preciso contar con un lenguaje dinámico y un entorno de programación integrado que permita realizar modificaciones en tiempo de ejecución, además de mecanismos de actualización adecuados para que todos los participantes experimenten un comportamiento similar del fenómeno observado, aún a pesar de estar utilizando equipos de diferente potencia o de estar interconectados por redes con distinto ancho de banda.

Para evaluar cada una de las características antes mencionadas, se ha experimentado con diferentes versiones de Open Cobalt. Ciertos aspectos como la fidelidad de la representación o la navegabilidad e interacción con el entorno se determinaron en forma directa, sin embargo para evaluar otros aspectos como los mecanismos de comunicación entre usuarios o la ejecución remota de aplicaciones externas, se instaló un servidor de aplicaciones y se realizaron pruebas con máquinas de diferente potencia conectadas en una LAN (Local Area Network) y a través de Internet. Por último, se implementaron simulaciones sencillas de fenómenos físicos (interacción gravitatoria) con la finalidad de evaluar no sólo el entorno de programación, sino también el comportamiento mismo de la simulación en un ambiente distribuido. A continuación, se presentan los resultados obtenidos.

\section{ANÁLISIS DE LOS RESULTADOS OBTENIDOS}

Fidelidad de la representación - Los espacios virtuales 3-D están compuestos principalmente por luces, texturas y modelos. A partir de un espacio completamente vacío, Open Cobalt permite crear un espacio virtual personalizado en forma muy sencilla por medio de la selección de texturas para el suelo y la bóveda celeste (SkyBox), la cual puede ser animada en forma independiente para brindar un mayor realismo a la escena. También es posible modificar las características de la luz ambiente y agregar las luces puntuales que se deseen.

Además, es posible agregar ciertos efectos como irregularidades en el terreno por medio de funciones fractales o un efecto de neblina a la escena. Es decir, el manejo de las texturas y de las luces, ya sean puntuales como de ambiente, que posee Open Cobalt permite un grado de realismo suficiente, aunque sin el grado de sofisticación de otros entornos, como Second Life o Blue Mars. Sin embargo, tanto la generación de terrenos irregulares, como la aplicación de efectos lumínicos y de niebla permiten crear mundos virtuales completamente diferentes de forma muy rápida y sencilla.

En la Figura 1 se aprecia una secuencia de imágenes que muestran diferentes etapas de la personalización de un espacio. Inicialmente se observa sólo al avatar dentro de un espacio vacío (A), luego se agrega la textura del suelo (B), posteriormente la textura de la bóveda celeste, la cuál puede ser animada para dar una apariencia más realista (C) y finalmente se agregan las montañas, las cuales son generadas por medio de algoritmos fractales (D).

Navegabilidad e interacción con el entorno - Navegar en un espacio virtual tridimensional suele producir cierto grado de desorientación si se carece de ciertas referencias que nos indiquen nuestra posición, la de los demás, la extensión del espacio, etc. y este efecto se hace más evidente al haber diferentes espacios virtuales conectados entre sí.

En Open Cobalt cuando un usuario se conecta a otro espacio virtual, automáticamente aparece en su espacio un "portal" por cada espacio con el que mantenga una conexión. Este portal no solo muestra, en tiempo real, lo que está sucediendo en el otro espacio sino que al atravesarlo se ingresa instantáneamente en dicho espacio. 


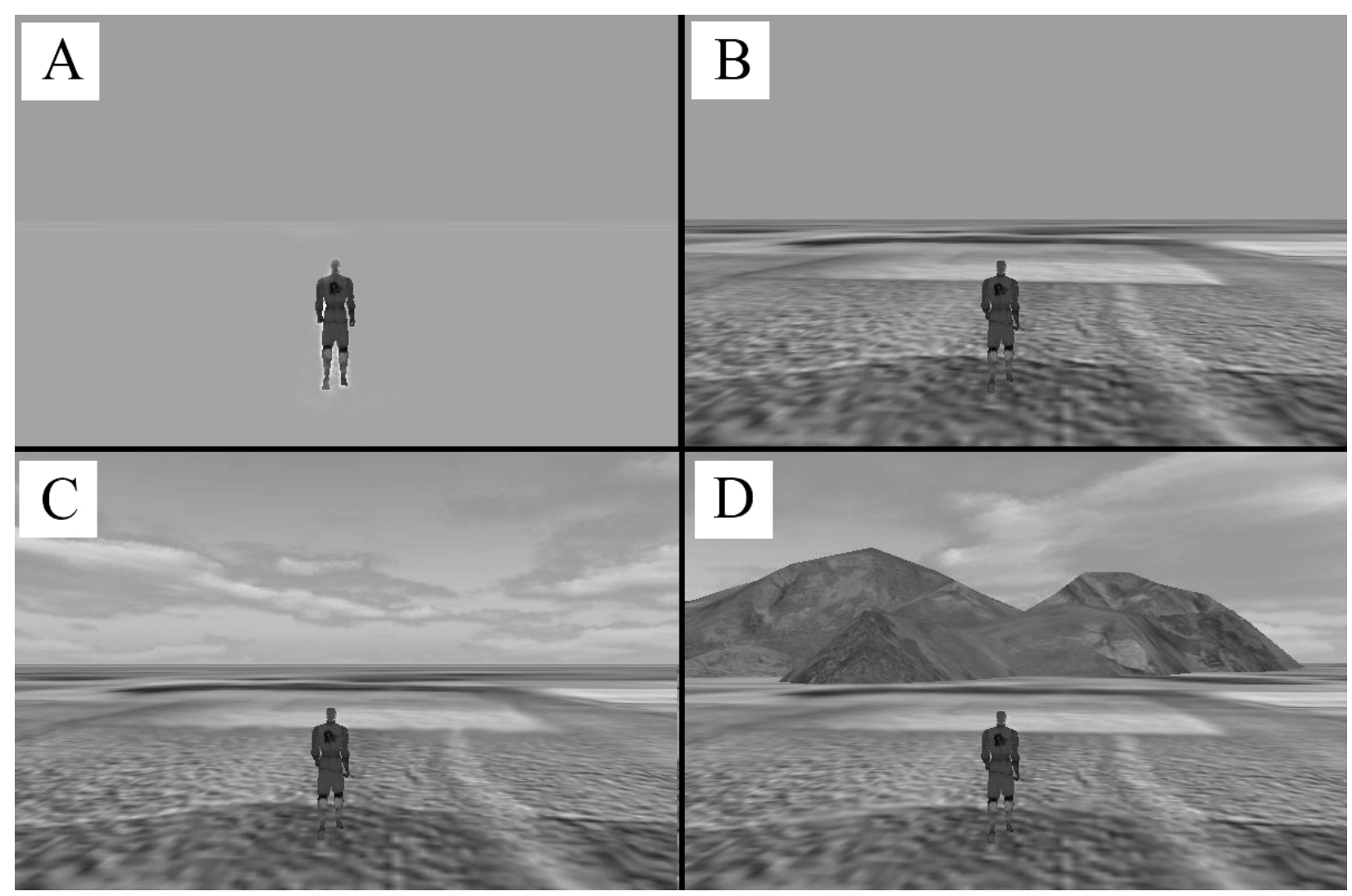

Fig. 1: Aplicación de luces, texturas y generación fractal del terreno.

Por lo tanto, ya que es posible estar conectado a varios espacios simultáneamente y cada usuario tiene la libertad de pasar de uno a otro simplemente atravesando el portal correspondiente, rápidamente aparece una cierta sensación de desorientación. Actualmente Open Cobalt cuenta con un mecanismo de "retorno de emergencia" que permite al usuario volver a su espacio original de partida. Sin embargo, sería deseable contar en el futuro con otras ayudas a la navegación, tales como visualización de la red de espacios interconectados, mapas de proximidad que indiquen la posición de otros usuarios, etc. Actualmente los desplazamientos de los avatars dentro de los espacios se comandan mediante dispositivos estándar como el mouse o el teclado, aunque algunos investigadores han realizado experiencias con otras interfaces como SmartBoards y Wiimote.

Comunicación entre usuarios - Las herramientas de comunicación son un elemento clave para lograr cualquier actividad de tipo colaborativa. En este sentido Open Cobalt proporciona dos opciones de comunicación textual, una local, para establecer comunicaciones dentro del mismo espacio y otra externa por medio del protocolo XMPP (Extensible Messaging and Presence Protocol), también conocido como Jabber, pudiendo utilizarse esta última a través de diferentes servidores gratuitos. Ambas opciones proporcionan un medio de comunicación claro y simple.

Para la comunicación oral se utiliza VolP (Voice over Internet Protocol) y el sonido 3-D permite establecer conversaciones con aquellos avatars que se encuentren en la cercanía. En las pruebas de conectividad realizadas dentro de una LAN (Local Area Network) funcionó adecuadamente, al igual que en varios de los encuentros semanales que mantuvimos con desarrolladores de la Universidad de Duke, a través de Internet. Por el momento, si bien es posible utilizar la animación de los avatars para desplazarse por el espacio virtual, no existe un repertorio de señas para comunicarse gestualmente y algunas funcionalidades del Chat, aún se encuentran en desarrollo.

Modelos 3-D y contenidos multimedia - Open Cobalt soporta la importación de diferentes formatos estándar de modelos 3-D, tales como .ase, .kmz, .obj y .vrml. En 2005, David Smith presentó una interfaz para crear y modificar modelos 3-D en Croquet (Smith y et al., 2005). Actualmente en Open Cobalt, si bien es posible importar modelos y avatars junto con la información necesaria 
para su animación, no es posible aún modificarlos, ni personalizarlos como en otras plataformas. Con respecto a otros contenidos multimedia es posible reproducir los formatos más extendidos y visualizar imágenes y videos en una ventana.

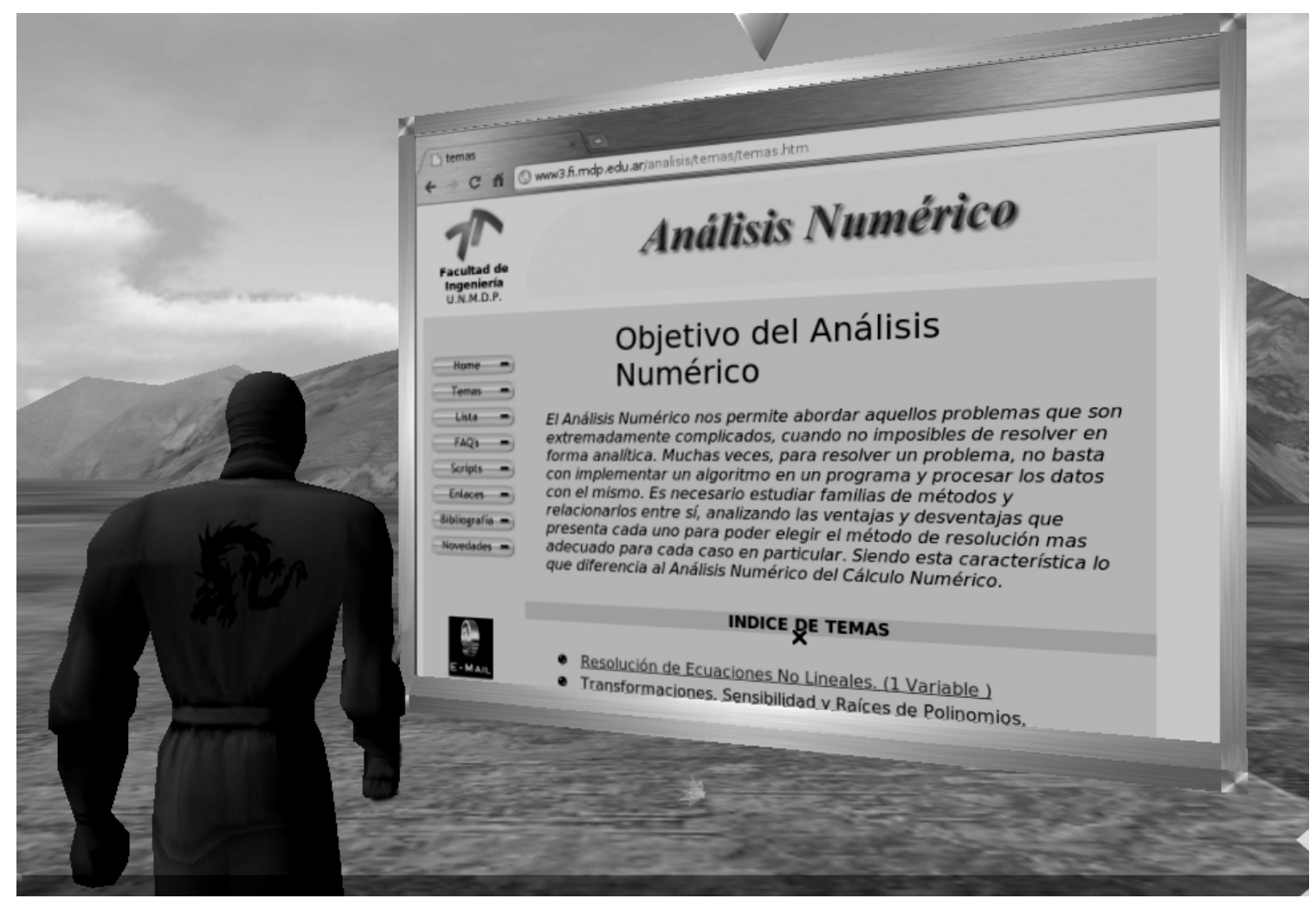

Fig. 2: Integración de aplicaciones externas al entorno virtual.

Integración de aplicaciones - Además de las herramientas propias del entorno, es posible ejecutar aplicaciones corrientes como navegadores web, procesadores de texto, planillas de cálculo, etc. provistas por un servidor VNC (Virtual Network Computing). Esto permite utilizar aplicaciones mono-usuario corrientes en un entorno multi-usuario colaborativo. Por ejemplo, varios estudiantes conectados al mismo espacio podrían escribir sobre el mismo documento utilizando el mismo procesador de textos, o utilizar cualquier otra aplicación, sin salir del entorno.

En la Figura 2 se observa la página web de nuestra asignatura, Análisis Numérico, a la cual se accede desde un navegador web (Firefox) directamente dentro del mismo entorno. En esta asignatura se estudian los fundamentos de varios métodos de resolución de ecuaciones diferenciales, los cuales son utilizados para simular diferentes fenómenos. De la misma manera es posible ejecutar aplicaciones gráficas para representar los resultados obtenidos, integrando así diferentes representaciones del fenómeno en estudio.

Entorno de programación - Squeak (Black et al. ,2007), no sólo es el lenguaje en el que está íntegramente desarrollado Open Cobalt sino que forma parte del mismo entorno y por lo tanto, todas las herramientas de programación y depuración dinámicas del ambiente son totalmente accesibles en el modo de programación. De esta manera es posible programar simulaciones numéricas que faciliten la comprensión de conceptos y fenómenos naturales relacionados con la ciencia y la ingeniería. Se ha demostrado que la utilización de simulaciones numéricas en el estudio de la mecánica de fluidos contribuye a aumentar el interés y la motivación del alumno, mejorando claramente su aprendizaje (Zamora y Kaiser, 2009).

Existen fenómenos físicos a nivel macroscópico o microscópico que son muy difíciles de reproducir y observar pero que pueden estudiarse simulando numéricamente las leyes que los gobiernan, dotando a los objetos del entorno de propiedades tales como masa o carga eléctrica y estudiar su comportamiento cuando están influenciados por campos gravitatorios, magnéticos, etc. 
(McCahill, 2006). En la Figura 3 se muestra una simulación gravitatoria a la cual se le ha integrado un modelo 3D de un satélite obtenido en forma libre y gratuita desde Google 3D Warehouse.

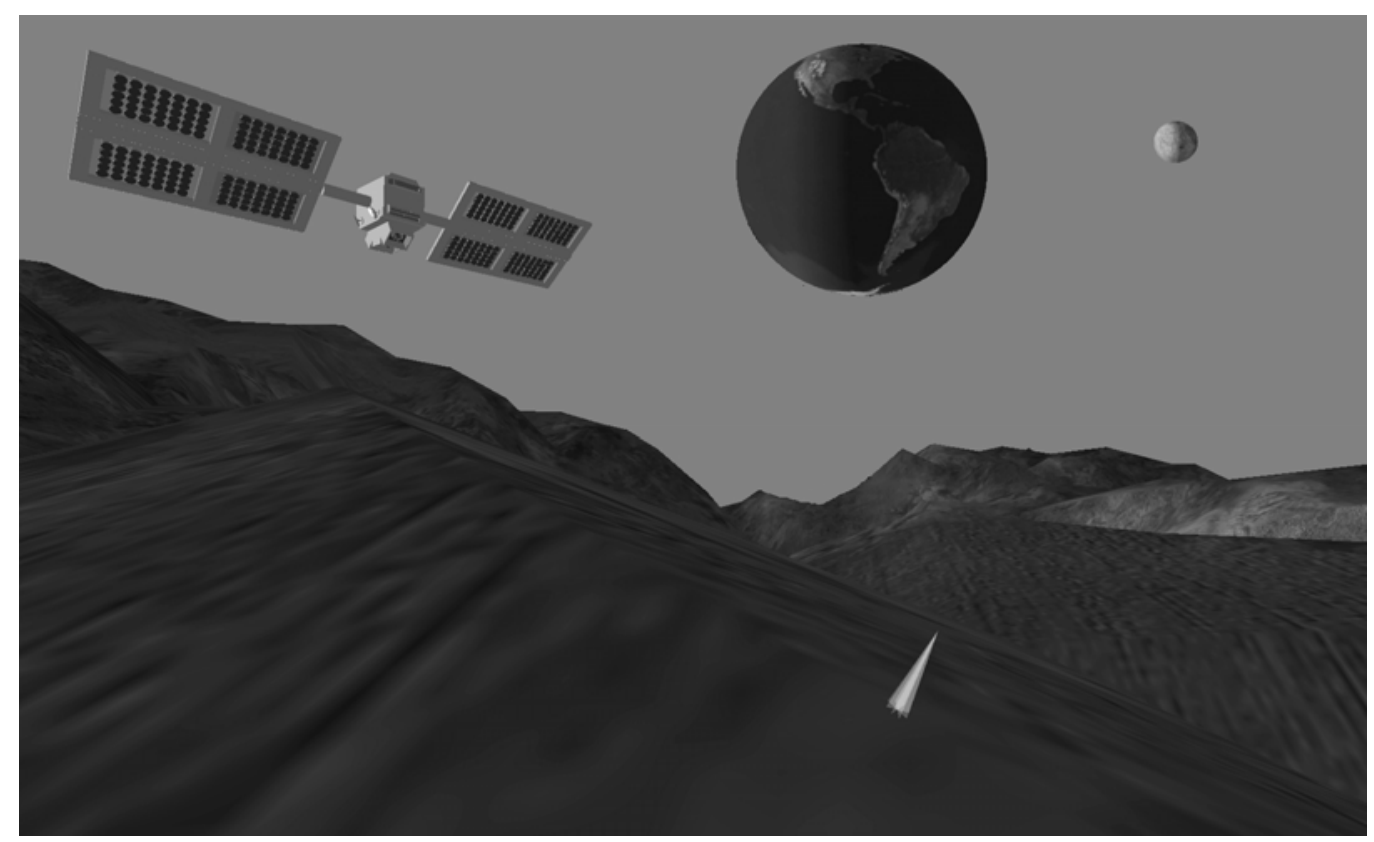

Fig. 3: Simulación gravitatoria e Integración de modelos 3D.

Por otra parte, en un entorno en el que se encuentran conectados varios estudiantes simultáneamente resulta crucial determinar si será posible observar un comportamiento idéntico del fenómeno en estudio en cada uno de los puestos de trabajo. Para analizar este punto, generamos un espacio virtual en el que incluimos una simulación sencilla de un fenómeno de atracción gravitacional y nos conectamos a dicho espacio con máquinas de diferente potencia. Lo que observamos es que aun cuando las actualizaciones de los cambios no eran simultáneas, los resultados observados por cada usuario por separado eran totalmente consistentes con el fenómeno.

\section{CONCLUSIONES}

A partir de los resultados obtenidos, se observa que es totalmente factible la implementación de simulaciones en un entorno virtual descentralizado como Open Cobalt, aunque lograr este cometido actualmente requiere de conocimientos de su funcionamiento interno y de programación en Smalltalk.

La utilización de librerías como OpenAl que produce el sonido espacial y OpenGL que realiza las operaciones matemáticas necesarias para la representación de texturas, luces, modelos 3D, etc. permiten la creación de simulaciones convincentes. Así como también, las herramientas de comunicación presentes en dicho entorno resultan adecuadas para coordinar tareas colaborativas.

Por otra parte, de las pruebas realizadas también surge la necesidad de contar con mecanismos más intuitivos de manipulación de objetos y con herramientas para la creación de modelos a partir de figuras geométricas primitivas. Otro aspecto a considerar es que actualmente las vistas son únicamente en primera persona. Es decir, la vista representada es la del propio avatar, por lo que sería conveniente contar con diferentes vistas y con otras formas de desplazamiento, además de mecanismos de ayuda a la navegación dentro de cada espacio y entre los diferentes espacios.

Es evidente que las experiencias obtenidas con Croquet (Stearns et. al, 2006), su antecesor, sin duda prepararon el terreno para Open Cobalt, aunque este último aún se encuentra en una fase alfa de su desarrollo. Probablemente aún sea necesario resolver ciertas cuestiones técnicas e incorporar mejoras en algunos aspectos, sin embargo, Open Cobalt ya cuenta con los componentes básicos necesarios para la creación de simulaciones virtuales con propósitos educativos. 


\section{REFERENCIAS}

Black A., Ducasse S., Nierstrasz O., Pollet D., Cassou D. y Denker M., Squeak by Example, $1^{\text {a }}$ edición, pp 287, Editorial Square Bracket Associates, Kehrsatz, Suiza, (2007)

Chun-Hong H. y Ming Z., Construction of Collaborative Virtual Learning Communities in Peer-topeer Networks, Intelligent Information Hiding and Multimedia Signal Processing, Vol.2, pp 305-308, (2008)

Fredes C., Hernández J. y Díaz D., Potencial y Problemas de la Simulación en Ambientes Virtuales para el Aprendizaje, Formación Universitaria, Vol. 5, №1, pp 45-56, (2012)

Girvan C. y Savage T., Identifying an appropriate pedagogy for virtual worlds: A Communal Constructivism case study, Computers \& Education, Vol. 55, pp 342-349, (2010)

Goldberg A. y Robson D., Smalltalk-80 The Language, $1^{\text {a }}$ edición, pp 581, Editorial Addison Wesley Publishing Co., Palo Alto, California, Estados Unidos, (1989)

Guzdial M., Squeak. Object Oriented Design with Multimedia Applications, $1^{\text {a }}$ edición, pp 306, Editorial Prentice Hall, Upper Saddle River, New Jersey, Estados Unidos, (2001)

Holmes, B., Tangney, B., Fitzgibbon, A., Savage, T. y Mehan, S., Communal Constructivism: students constructing learning for as well as with others, Proceedings of the 12th International Conference of the Society for Information Technology and Teacher Education (SITE 2001), pp 3114-3119, Orlando, Florida, Estados Unidos, 5 al 10 de Marzo, (2001)

Huang H., Rauch U. y Liaw S., Investigating learners' attitudes toward virtual reality learning environments: Based on a constructivist approach, Computers\&Education, Vol. 55, pp 1171-1182, (2010)

Ingalls, D., Kaehler, T., Maloney, J., Wallace, S., y Kay, A., Back to the Future: The Story of Squeak. A Practical Smalltalk Written in Itself, SIGPLAN Not., Vol. 32, Nro. 10, pp 318-326, (1997)

Kypuros, J. y Connolly, T., Collaborative Experimentation and Simulation: A Pathway to Improving Student Conceptualization of the Essentials of System Dynamics and Control Theory, Proceedings of the 2005 American Society for Engineering Education Annual Conference \& Exposition, NSF Grantees Poster Session, pp 1-11, Portland, Oregon, Estados Unidos, 12 al 15 de Junio, (2005)

Leask M. y Younie S., Communal constructivist theory: information and communications technology pedagogy and internationalisation of the curriculum, Journal of Information Technology for Teacher Education, Vol. 10:1-2, pp 117-134, (2001)

Lombardi J. y Lombardi M., Opening the Metaverse, Online Worlds: Convergence of the Real and the Virtual, $1^{\mathrm{a}}$ edición, Editorial Springer-Verlag Human Computer Interaction Series, pp 111-122, Londres, Inglaterra, (2010)

McCahill, M., Moore, P., Wendland L. y Zampogna, A., Extending Croquet Spaces with Vector Fields, Vehicles and Virtual Presence, Proceedings of the Fourth International Conference on Creating, Connecting and Collaborating through Computing, IEEE Computer Society, pp 68-72, Berkeley, California, Estados Unidos, 26 a 27 de Enero, (2006)

McGeer, R., Raab, A., Reed, D., Smith D. y Kay, A. Scalability of Collaborative Environments. Proceedings of the Fourth International Conference on Creating, Connecting and Collaborating through Computing, IEEE Computer Society, pp 168-174, Berkeley, California, Estados Unidos, 26 a 27 de Enero, (2006) 
Open Cobalt License (2008) - MIT open source license. http://www.opencobalt.org/license. Acceso: 29 de Mayo (2012)

Smith, D., Kay, A., Raab, A. y Reed, D. Croquet. A Collaboration System Architecture, Proceedings of the First International Conference on Creating, Connecting and Collaborating through Computing, IEEE Computer Society, pp 2-9, Heian Jingu Shrine, Kyoto, Japón, 31 de Enero, (2003)

Smith, D., Raab, A., Ohshima, Y., Reed, D., y Kay, A., Filters and Tasks in Croquet, Proceedings of the Third International Conference on Creating, Connecting and Collaborating through Computing, IEEE Computer Society, pp 50-56, Cambridge, Massachusetts, Estados Unidos, 28 a 29 de Enero, (2005)

Sproull B., Alan Kay a visionary designer, Points of View: a tribute to Alan Kay, $1^{\text {a }}$ edición, Editorial Typecraft Wood \& Jones Inc., pp 1-4, Pasadena, California, Estados Unidos, (2010)

Stearns, H., Gargus, J., Schuetze, M. y Lombardi, J., Simplified Distributed Authoring Via Component-based Object Construction and Deconstruction in Collaborative Croquet Spaces, Proceedings of the Fourth International Conference on Creating, Connecting and Collaborating through Computing, IEEE Computer Society, pp 79-87, Berkeley, California, Estados Unidos, 26 a 27 de Enero, (2006)

Warburton, S., Second Life in higher education: Assessing the potential for and the barriers to deploying virtual worlds in learning and teaching, British Journal of Educational Technology, Vol. 40, No. 3, pp 414-426, (2009)

Zamora, B. y Kaiser, A., Enseñanza de Temas Avanzados de Mecánica de Fluidos usando Dinámica de Fluidos Computacional, Formación Universitaria, Vol. 2, №1, pp 27-36, (2009) 